\title{
VLT/X-Shooter survey of near-infrared diffuse interstellar bands ${ }^{\star}$
}

\author{
N. L. J. Cox ${ }^{1}$, J. Cami ${ }^{2,3}$, L. Kaper ${ }^{4}$, P. Ehrenfreund ${ }^{5}$, B. H. Foing ${ }^{6}$, B. B. Ochsendorf ${ }^{7}$, \\ S. H. M. van Hooff ${ }^{4}$, and F. Salama ${ }^{8}$ \\ ${ }^{1}$ Instituut voor Sterrenkunde, KU Leuven, Celestijnenlaan 200D, Bus 2401, 3001 Leuven, Belgium \\ e-mail: nick.cox@ster . kuleuven . be \\ 2 Department of Physics and Astronomy, The University of Western Ontario, London, ON N6A 3K7, Canada \\ 3 SETI Institute, 189 Bernardo Ave, Suite 100, Mountain View CA 94043, USA \\ ${ }^{4}$ Sterrenkundig Instituut Anton Pannekoek, Universiteit van Amsterdam, Science Park 904, 1098 Amsterdam, The Netherlands \\ 5 Space Policy Institute, 1957 E Street, 20052 Washington DC, USA \\ ${ }^{6}$ ESTEC, European Space Agency, Keplerlaan 1, 2201 AZ Noordwijk ZH, The Netherlands \\ 7 Leiden Observatory, Leiden University, Niels Bohrweg 2, 2333 CA Leiden, The Netherlands \\ 8 NASA AMES Research Centre, CA, USA
}

Received 15 November 2013 / Accepted 2 July 2014

\section{ABSTRACT}

\begin{abstract}
Context. The unknown identity of the diffuse interstellar band (DIB) carriers poses one of the longest standing unresolved problems in astrophysics. While the presence, properties, and behaviour of hundreds of optical DIBs between $4000 \AA$ and $9000 \AA$ have been well established, information on DIBs in both the ultra-violet and near-infrared (NIR) ranges is limited.

Aims. In this paper, we present a spectral survey of the NIR range, from $0.9 \mu \mathrm{m}$ to $2.5 \mu \mathrm{m}$. Our observations were designed to detect new DIBs, confirm previously proposed NIR DIBs, and characterise their behaviour with respect to known line-of-sight properties (including the optical DIBs present in our spectra).

Methods. Using the X-Shooter instrument mounted on the ESO Very Large Telescope (VLT) we obtained medium-resolution spectra of eight known DIB targets and one telluric reference star, from $3000 \AA$ to $25000 \AA$ in one shot.

Results. In addition to the known 9577, 9632, 10780, 11797, and $13175 \AA$ NIR DIBs, we confirm 9 out of the 13 NIR DIBs that were presented by Geballe et al. (2011, Nature, 479, 200). Furthermore, we report 11 new NIR DIB candidates. The strengths of the strongest NIR DIBs show a general correlation with reddening, $E_{(B-V)}$, but with a large scatter. Several NIR DIBs are more strongly correlated with the $5780 \AA$ DIB strength than with $E_{(B-V)}$; this is especially the case for the $15268 \AA$ DIB. The NIR DIBs are strong: the summed equivalent widths of the five strongest NIR DIBs represent a small percent of the total equivalent width of the entire average DIB spectrum (per unit reddening). The NIR DIBs towards the translucent cloud HD 147889 are all weak with respect to the general trend. No direct match was found between observed NIR DIBs and laboratory matrix-isolation spectroscopic data of polycyclic aromatic hydrocarbons (PAHs).

Conclusions. The strong correlation between the 5780-15268 DIB pair implies that $(N f)_{5780} /(N f)_{15268}=14$. However, the reduced strength of the $15268 \AA$ DIB in HD 147889 rules out a common carrier for these two DIBs. Since the ionisation fraction for small PAHs in this translucent cloud is known to be low compared to diffuse clouds, the weakness of the $15268 \AA$ DIB suggests that an ionised species could be the carrier of this NIR DIB.
\end{abstract}

Key words. ISM: lines and bands - dust, extinction - line: identification - line: profiles

\section{Introduction}

Diffuse interstellar bands (DIBs) are observed throughout the Universe, and represent a major, albeit unidentified, fraction of interstellar matter in the Milky Way and beyond (e.g. Snow 2002; Cordiner 2014, and references therein). Several hundred strong and weak DIBs have been reported between $4000 \AA$ and 9000 A (Jenniskens \& Désert 1994; Tuairisg et al. 2000; Hobbs et al. 2009), while only a few are known in the NIR longwards of $9000 \AA$ (Joblin et al. 1990; Foing \& Ehrenfreund 1994; Geballe et al. 2011). Despite intensive study - and debate - of a number of propositions and reported laboratory matches, not a single DIB carrier has been unambiguously identified. For a review of the various proposed candidate carriers, see the seminal work by Herbig (1995); Sarre (2006) provides a detailed discussion of more recent developments in the quest for the DIB carriers.

\footnotetext{
* Based on observations obtained with the VLT (ESO Programme 385.C-0720).
}

One important question arising from DIB studies is whether DIB features are also present at shorter ultra-violet (UV) and longer IR wavelengths. The presence/absence of DIBs in the UV and IR is important for testing the PAH-DIB hypothesis (Salama et al. 1996; Ehrenfreund et al. 1995; Cox 2011), because charged polycyclic aromatic hydrocarbons (PAHs) containing more than about 20 carbon atoms are known to have their main electronic transitions in the NIR range, whereas neutral PAHs and small charged PAHs absorb predominantly at ultra-violet and visible wavelengths, respectively (see Tielens 2008). Salama et al. (2011) and Gredel et al. (2011) searched for the main transition of neutral PAHs in astronomical optical spectra, but no matches were found and only upper limits could be set to their abundance. On the other hand, PAHs containing more than 40 carbon atoms are quite stable, could be fully hydrogenated (Vuong \& Foing 2000), and have strong transitions in the (near-)infrared. Hence, NIR spectroscopic (DIB) surveys may reveal their presence in the interstellar medium (ISM). Several ongoing and 
Table 1. Observed targets.

\begin{tabular}{lllllll}
\hline \hline Target & $\begin{array}{l}\text { Spectral } \\
\text { type }^{a}\end{array}$ & $\begin{array}{l}B-V \\
(\mathrm{mag})\end{array}$ & $\begin{array}{l}(B-V)_{0}{ }^{b} \\
(\mathrm{mag})\end{array}$ & $\begin{array}{l}E_{(B-V)}{ }^{c} \\
(\mathrm{mag})\end{array}$ & $\begin{array}{l}A_{V} \\
(\mathrm{mag})\end{array}$ & $\begin{array}{l}v_{\text {shift }^{d}} \\
\left(\mathrm{~km} \mathrm{~s}^{-1}\right)\end{array}$ \\
\hline HD 167785 & B2 V & -0.09 & $-0.21^{1},-0.24^{2}$ & 0.14 & $0.42(0.05)$ & -7.0 \\
HD 326306 & B1 V & -0.11 & $-0.23^{1},-0.26^{2}$ & 0.14 & $0.42(0.05)$ & 8.0 \\
HD 153294 & B7 Ib/II & +0.03 & $-0.04^{1},-0.12^{2}$ & $0.07,0.15$ & $0.22-0.47$ & 14.0 \\
HD 152246 & O9 Ib & +0.12 & $-0.27^{1},-0.28^{2}$ & $0.39(0.03)$ & $1.22(0.03)$ & 6.5 \\
HD 161056 & B1.5 V & +0.38 & $-0.22^{1},-0.25^{2}$ & $0.62(0.05)$ & $1.90(0.05)$ & -3.5 \\
HD 161061 & O $^{+}$ & +0.62 & $-0.30^{1},-0.29^{2}$ & $0.92(0.03)$ & $2.83(0.04)$ & 7.5 \\
HD 147889 & B2 III/IV & +0.71 & $-0.19^{1},-0.24^{2}$ & $0.90(0.06)$ & $3.7(0.1)$ & 10.0 \\
HD 183143 & B7 Iae & +1.00 & $-0.04^{1},-0.04^{2}$ & $1.04(0.03)$ & $3.97(0.05)$ & -5.0 \\
4U 1907+09 & O9 Ia & +3.2 & $-0.28^{1},-0.28^{2}$ & $3.48(0.03)$ & $10.9(0.05)$ & 25.0 \\
\hline
\end{tabular}

Notes. ${ }^{(a)}$ Spectral types taken from the Simbad database, except for 4U 1907+09 taken from Cox et al. (2005) and HD 161061 taken from Mazzei $\&$ Barbaro (2011). ${ }^{(b)}$ Intrinsic colours from (1) Wegner (1994) and (2) Fitzgerald (1970). ${ }^{(c)} E_{(B-V)}$ values are derived from $B$ and $V$ magnitudes (Simbad) and intrinsic colours, $(B-V)_{0}$. We took the mean of the intrinsic colours from (1) and (2) with uncertainties including inaccuracy, by one step, in Morgan-Keenan spectral-luminosity classification. We adopt $E_{(B-V)}=0.10$ mag for HD 153294. $A_{V}=R_{V} \times E_{(B-V)}$. We adopt $R_{V}=3.1$ except for $\mathrm{HD} 147889\left(R_{V}=4.1\right)$ and $\mathrm{HD} 183143\left(R_{V}=3.8\right)$. Uncertainties in parentheses. ${ }^{(d)}$ The heliocentric radial velocity of the strongest components in the atomic line of KI $\left(\lambda_{\text {rest }}=7698.974 \AA\right)$ used to shift the spectra to the respective "LSR" rest wavelengths. Corrections were verified for consistency using additional interstellar lines of the Na I D doublet, $\mathrm{CH}\left(\lambda_{\text {rest }}=4300.303 \AA\right)$, and $\mathrm{CH}^{+}\left(\lambda_{\text {rest }}=4232.548 \AA\right)$.

future large NIR spectroscopic surveys include NIR DIBs and hence an accurate catalogue of these features, including an understanding of their behaviour with respect to other interstellar features, is warranted. For example, Zasowski \& Ménard (2014) and Zasowski et al. (2014) present first results on two NIR DIBs in SDSS-III/APOGEE spectra and their Galactic distribution. Notwithstanding this potential importance, the NIR wavelength range $(0.9-2.5 \mu \mathrm{m})$ has so far received little attention. Adamson et al. (1994) have searched, without success, for the $13175 \AA$ band in dense environments. Geballe et al. (2011) presented the discovery of a dozen NIR DIB candidates longwards of $1.5 \mu \mathrm{m}$. These new finding have revived interest for this spectral range in context of the properties and chemical complexity of the ISM in general, and DIB carriers in particular. Searches for DIBs in the near-UV at wavelengths shortwards of $3300 \AA$ have thus far shown that there are apparently far fewer DIBs, if any, in the near-UV than in the optical (Gnacinski et al. 1997; Watson 2001; Bhatt et al., in prep.).

This paper presents the results of an exploratory VLT/X-Shooter survey of NIR DIBs in the spectra of a sample of reddened early-type stars. This works seeks to confirm and extend the results on the presence, properties and behaviour of NIR DIBs in diffuse/translucent interstellar clouds. The observations and data reduction are briefly discussed in Sect. 2. Then, in Sect. 3 we present spectra, equivalent width and full-width-at-half-maximum (FWHM) measurements of known visible and NIR DIBs; we also identify additional NIR DIBs. The NIR DIB properties in terms of strength and width are discussed in Sect. 4 together with correlations of DIB strengths with dust (reddening) and with other DIBs. In Sect. 5 the observations are compared with previous laboratory measurements. Section 6 discusses the measured DIB properties for the translucent cloud towards HD 147889 in context of the expected PAH population. The main conclusions of this survey are summarised in Sect. 7.

\section{Optical and NIR spectroscopy with VLT/X-Shooter}

We selected a small sample of bright reddened early-type stars whose lines of sight give rise to a range in magnitudes of ex- tinction. The diffuse clouds in these sightlines are also exposed to the interstellar radiation field in varying degrees. This optimises the ability to detect new NIR DIBs and study their behaviour in the ISM. The observed targets are listed in Table 1 with their interstellar reddening, $E_{(B-V)}$, visual extinction, $A_{V}$, and spectral type. To obtain a spectroscopically unbiased view of NIR DIBs, spectra were obtained with X-Shooter, a wide-band intermediate-resolution spectrograph at the ESO Very Large Telescope (D'Odorico et al. 2006; Vernet et al. 2011). X-Shooter has three separate arms (optical light paths) - UVB, VIS, and NIR - each with their own optimised optical and system design. In this way it is possible to obtain in one shot a nearly contiguous spectrum from $3000 \AA$ to $24800 \AA$. The spectra were reduced, separately for each arm, using the esorex X-Shooter pipeline (Modigliani et al. 2010). Standard parameters were adopted for preparing the calibration files. The final science spectra were reduced using non-default stricter cosmic ray hit rejection criteria which significantly improved the final spectra by removing spurious glitches. In this paper we focus on the NIR spectra which cover the range from $9940 \AA$ to $24790 \AA$. A $0.4^{\prime \prime}$ slit, with a fixed length of 11 ", was used in the "Auto Nod on Slit Mode" yielding a spectral resolving power of $\sim 10000$ in the NIR arm. This corresponds to an instrumental FWHM, measured from telluric lines, of $\sim 1.3 \AA$ in the range $1.1-1.3 \mu \mathrm{m}, \sim 1.7 \AA$ in the range $1.5-1.7 \mu \mathrm{m}$, and $\sim 1.9 \AA$ at $1.8 \mu \mathrm{m}^{1}$. The telluric correction tool Spextool (see Vacca et al. 2003 and Cushing et al. 2004) was used to remove atmospheric absorption lines from the observed NIR spectra by way of dividing the spectra with a telluric standard spectrum. In order to have an independent verification of the procedure and the final corrected spectrum we use both a computed atmospheric model and the spectrum of an unreddened B star. The hydrogen lines are removed from the telluric standard as part of the Spextool telluric correction procedure. The telluric correction is prone to introduce significant residuals in regions with dense forests of strong telluric absorption lines. The following regions are most severely affected: $13500-14800 \AA$, $17900-19000 \AA$, 19950-20250 A, 20 500-20750 ̊, $23150-24800 \AA$. However, even in those

\footnotetext{
1 All quoted rest wavelengths are those measured in air unless otherwise specified. DIB rest wavelengths given by Geballe et al. (2011) are in vacuum.
} 

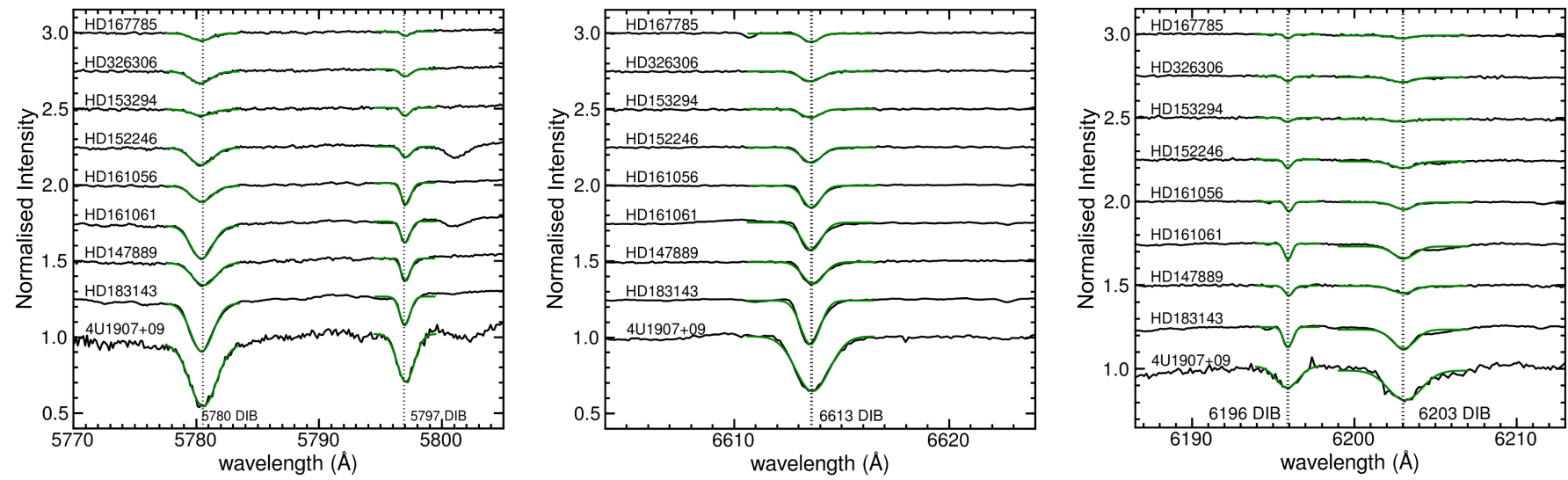

Fig. 1. Strong DIBs at $5780 \AA$ and $5797 \AA$ (left), $6613 \AA$ (middle), and 6196 and $6203 \AA$ (right) are shown for the observed targets. Solid green curves represent the Gaussian model fit to the data.

cases the narrow residuals can sometimes be distinguished from intrinsic stellar and interstellar lines.

\section{Diffuse interstellar bands}

\subsection{Measurements of optical diffuse interstellar bands}

Before studying the NIR DIBs, we measured the equivalent widths of several strong DIBs in the optical range (X-Shooter VIS arm; see Fig. 1 and Table 2). The average equivalent width per unit reddening for the seven strong DIBs at 5780, 5797, $5849,6196,6203,6270$, and $6613 \AA$ is $1.6 \AA / E_{(B-V)}$.

We also used $\mathrm{Na} I$ and $\mathrm{K} I$ absorption lines to determine the radial structure of the ISM, providing the opportunity to shift the observed optical and NIR DIBs to a common restframe. Once applied, this correction ensures that wavelength positions of the interstellar lines coincide between different lines of sight, while telluric and stellar lines display a shift due to the radial velocity with respect to the ISM.

\subsection{Detection of NIR diffuse interstellar bands}

Joblin et al. (1990) reported the first two NIR DIBs at $11797 \AA$ and $13175 \AA$ towards several reddened Galactic lines of sight. Next, the NIR DIBs at $9577 \AA$ and $9632 \AA$ were detected in a larger sample of bright OB stars (Foing \& Ehrenfreund 1994; Galazutdinov et al. 2000). As part of their spectral atlas of OB stars Groh et al. (2007) reported two unidentified features at 10780 and $10792 \AA$ in 9 lines of sight. The absorption band at $10780 \AA$ was found to correlate well with reddening (cf. their Fig. 4). Recently, Geballe et al. (2011) reported the detection of 13 new NIR DIBs, measured towards the Galactic Centre, 4 of which are also present in the average spectrum of the heavily reddened Cygnus OB2 sightlines. Figures 2 to 5 exhibit most of these NIR DIBs in our sample of low- to moderately-high reddening lines of sight. The spectra have been corrected for heliocentric motion as well as for the radial velocity of the main interstellar cloud in the line-of-sight according to the optical atomic absorption lines of $\mathrm{Na}$ I and K I (Sect. 3.1).

Owing to the limited signal-to-noise ratio and intrinsic weakness most of the known NIR DIBs are only detected in the three lines of sight with a visual extinction above 2 mag (i.e. HD 161061, HD 183143, 4U 1907+09). The strong and narrow $\lambda \lambda 11797,13175,15268$ DIBs are detected in some other

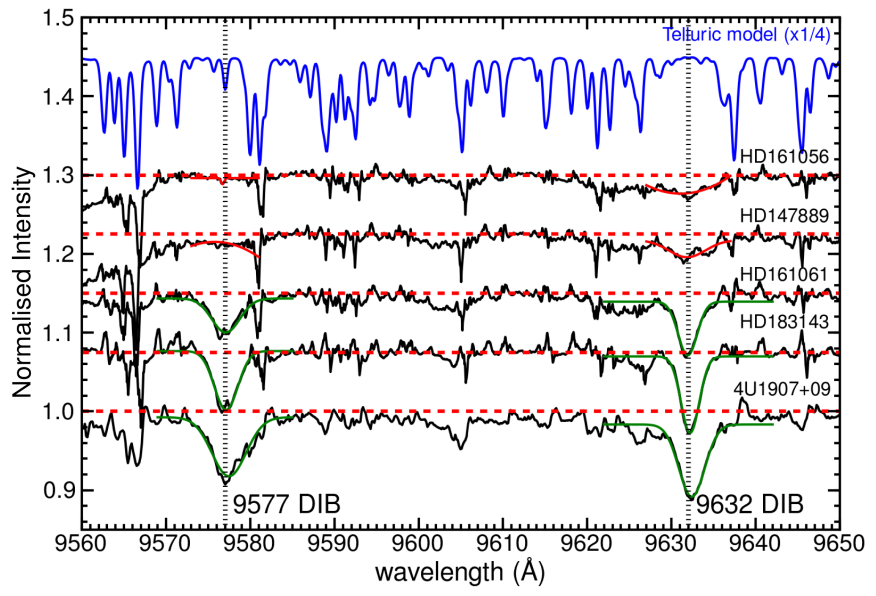

Fig. 2. NIR DIBs at $9577 \AA$ and $9632 \AA$ (assigned to the fullerene cation $\mathrm{C}_{60}^{+}$by Foing \& Ehrenfreund 1994) are shown for the reddened $\mathrm{X}$-Shooter sample of sightlines. The spectra have been corrected for the radial velocity of the ISM (including heliocentric correction). The vertical dotted lines indicate the rest wavelength for the respective DIBs. Dashed horizontal lines correspond to the normalised continua. Spectra are vertically offset for clarity. The atmospheric model spectrum (blue) is shown at the top (normalised and vertically compressed) in order to appreciate and evaluate the telluric correction. Solid green curves show the Gaussian model fits. The weak feature at $9633 \AA$ towards HD 161056 and HD 147889 indicates the presence of a weak stellar Mg II line. This has been noted before by Foing \& Ehrenfreund (1997) and estimated by Galazutdinov et al. (2000) to have an equivalent width of $50 \mathrm{~m} \AA$ for spectral type B2 III and $80 \mathrm{~m} \AA$ for B7 I. These authors assigned two additional features at $9603 \AA$ and $9625 \AA$, present also in our spectra, to stellar He I.

sightlines as well. Of the thirteen new NIR DIBs reported in Geballe et al. (2011) we detect three in three or more sightlines. Three others are detected in at least two sightlines while another three are detected only towards 4U 1907. Four NIR DIBs reported by Geballe and co-workers could not be confirmed (see below). The presence and absence of NIR DIBs in this XShooter survey is summarised as follows:

- The NIR DIBs at 9577 and $9632 \AA$ (Foing \& Ehrenfreund 1994) are detected in three lines of sight (Fig. 2).

- The NIR DIB at $10780 \AA$ (Groh et al. 2007) is detected in three lines of sight (Fig. 3). 


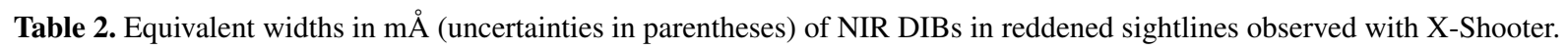

\begin{tabular}{|c|c|c|c|c|c|c|c|c|c|}
\hline$\lambda_{\mathrm{DIB}}{ }^{a}$ & HD 167785 & HD 326306 & HD 153294 & HD 152246 & HD 161056 & HD 161061 & HD 147889 & HD 183143 & $4 U 1907$ \\
\hline $\begin{array}{l}5780 \\
5797 \\
5849 \\
6196 \\
6203 \\
6270 \\
6613 \\
\end{array}$ & $\begin{array}{l}98(4) \\
26(2) \\
5(3) \\
13(1) \\
26(3) \\
17(3) \\
50(3)\end{array}$ & $\begin{array}{l}161(6) \\
53(3) \\
19(2) \\
19(1) \\
51(3) \\
37(2) \\
79(2)\end{array}$ & $\begin{array}{l}90(7) \\
48(2) \\
22(2) \\
17(2) \\
30(4) \\
14(2) \\
62(2)\end{array}$ & $\begin{array}{l}253(8) \\
75(3) \\
21(1) \\
30(1) \\
73(6) \\
50(3) \\
115(2)\end{array}$ & $\begin{array}{l}220(5) \\
135(3) \\
59(1) \\
32(1) \\
62(2) \\
52(2) \\
152(2)\end{array}$ & $\begin{array}{l}486(9) \\
133(4) \\
50(2) \\
56(2) \\
122(8) \\
100(4) \\
207(5)\end{array}$ & $\begin{array}{l}349(8) \\
137(3) \\
74(2) \\
40(2) \\
71(3) \\
54(6) \\
177(4)\end{array}$ & $\begin{array}{l}742(12) \\
197(6) \\
61(2) \\
87(2) \\
202(9) \\
170(5) \\
329(5)\end{array}$ & $\begin{array}{l}1172(31) \\
540(16) \\
186(10) \\
218(21) \\
465(26) \\
405(12) \\
703(13)\end{array}$ \\
\hline $\begin{array}{l}9577 \\
9632 \\
10780 \\
11797 \\
13175 \\
15268 \\
15610 \\
15646 \\
15666 \\
16227 \\
16567 \\
16578 \\
16588 \\
17803\end{array}$ & $\begin{array}{l}- \\
- \\
- \\
- \\
- \\
54(14) \\
- \\
- \\
- \\
- \\
- \\
- \\
- \\
-\end{array}$ & $\begin{array}{l}- \\
- \\
- \\
21(7) \\
53(12) \\
93(15) \\
- \\
- \\
- \\
- \\
- \\
- \\
- \\
-\end{array}$ & $\begin{array}{l}- \\
- \\
- \\
- \\
- \\
60(16) \\
- \\
- \\
- \\
- \\
- \\
- \\
- \\
- \\
-\end{array}$ & $\begin{array}{l}- \\
- \\
- \\
27(8) \\
40(24) \\
146(18) \\
- \\
- \\
- \\
- \\
- \\
- \\
- \\
-\end{array}$ & $\begin{array}{l}- \\
- \\
- \\
- \\
- \\
85(19) \\
- \\
- \\
- \\
- \\
- \\
- \\
- \\
-\end{array}$ & $\begin{array}{l}180(5) \\
195(4) \\
119(6) \\
145(7) \\
419(33) \\
231(17) \\
79(30) \\
- \\
- \\
- \\
- \\
- \\
- \\
-\end{array}$ & $\begin{array}{l}\leq 90 \\
\leq 120 \\
- \\
56(17) \\
203(53) \\
\approx 40(7) \\
- \\
- \\
- \\
- \\
- \\
- \\
- \\
-\end{array}$ & $\begin{array}{l}260(5) \\
263(3) \\
148(8) \\
110(9) \\
493(36) \\
326(28) \\
116(43) \\
153(88) \\
78(10) \\
- \\
- \\
30(10) \\
- \\
-\end{array}$ & $\begin{array}{l}380(6) \\
360(5) \\
181(11) \\
224(12) \\
819(36) \\
554(38) \\
189(66) \\
174(57) \\
188(15) \\
696(148) \\
37(6) \\
53(8) \\
18(5) \\
502(166)\end{array}$ \\
\hline $\begin{array}{l}10361 \\
10393 \\
10438 \\
10504^{c, d} \\
10507^{c, d}\end{array}$ & $\begin{array}{l}- \\
- \\
- \\
- \\
- \\
-\end{array}$ & $\begin{array}{l}- \\
- \\
- \\
- \\
- \\
-\end{array}$ & $\begin{array}{l}- \\
- \\
- \\
- \\
- \\
-\end{array}$ & $\begin{array}{l}- \\
- \\
- \\
- \\
- \\
-\end{array}$ & $\begin{array}{l}- \\
- \\
- \\
- \\
- \\
-\end{array}$ & $\begin{array}{l}18(4) \\
25(8) \\
28(4) \\
36 \\
29 \\
(66)^{b}\end{array}$ & $\begin{array}{l}- \\
- \\
- \\
- \\
- \\
(295)^{b}\end{array}$ & $\begin{array}{l}46(32) \\
19(5) \\
56(9) \\
58 \\
261 \\
(203)^{b}\end{array}$ & $\begin{array}{l}69(10) \\
56(9) \\
65(15) \\
136 \\
68\end{array}$ \\
\hline $\begin{array}{l}10697^{d} \\
13027^{c}\end{array}$ & $\begin{array}{l}- \\
-\end{array}$ & - & $\begin{array}{l}- \\
-\end{array}$ & $\begin{array}{l}- \\
-\end{array}$ & - & $\begin{array}{l}127(19) \\
71(15)\end{array}$ & - & $\begin{array}{l}262(36) \\
171(37)\end{array}$ & $\begin{array}{l}329(22) \\
222(41)\end{array}$ \\
\hline
\end{tabular}

Notes. ${ }^{(a)}$ Measured rest peak wavelengths in air are $9577.1 \pm 0.2,9632.0 \pm 0.2,10780.3 \pm 0.2,11797.0 \pm 0.5,13175.0 \pm 0.5,15267.8 \pm 0.4$, $15610.0 \pm 0.5,15645.5 \pm 0.5,15665.5 \pm 0.5,16226.7 \pm 0.5,16567.0 \pm 0.5,16578.0 \pm 0.5,16588.0 \pm 0.5,17802.7 \pm 0.5,10360.5 \pm 0.3$, $10392.9 \pm 0.3,10438.4 \pm 0.1,10504.2 \pm 0.2,10506.6 \pm 0.2,10696.9 \pm 0.2,13026.7 \pm 0.5 \AA$. (b) Integrated equivalent width for blended "W-shape" features at $10504 \AA$ \& $10506 \AA$. Absorption lines for HD 183143 are contaminated by a strong emission feature. ${ }^{(c)}$ Complex profile; possible blended lines. ${ }^{(d)}$ No sensible uncertainties could be determined for individual components.

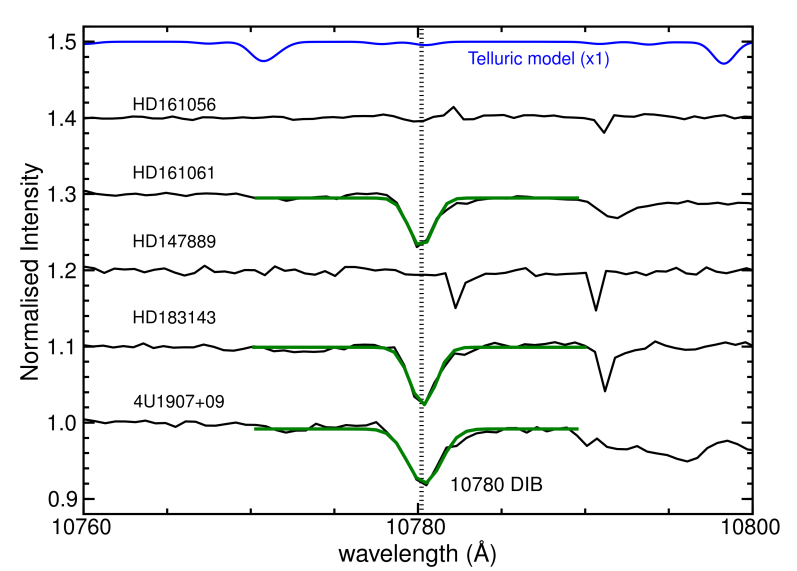

Fig. 3. NIR DIBs at $10780 \AA$ (Groh et al. 2007) are shown for the reddened X-Shooter sample of sightlines. The spectra have been corrected for the presence of telluric lines and subsequently for the radial velocity of the ISM. The dashed lines indicate the rest wavelength for the respective DIB. Solid green curves show the Gaussian model fits and the blue solid curve displays the telluric model spectrum.

- The NIR DIBs at 11797 and $13175 \AA$ (Joblin et al. 1990) are detected in seven lines of sight (Fig. 4).
- The strong NIR DIB at $\lambda_{\text {rest }} 15267.8 \AA$ reported by Geballe et al. (2011) is detected in nine sightlines (Fig. 4).

- The bands at $\lambda_{\text {rest }} 15610$ and $15665 \AA$ (Geballe et al. 2011) are detected in three lines of sight (Fig. 5).

- The $\lambda_{\text {rest }} 15646$ and $16588 \AA$ AIBs (Geballe et al. 2011) are seen in two sightlines (Fig. 5).

- The blended NIR DIB complex at 16560-16590 ̊ reported by Geballe et al. (2011) is resolved into three separate components.

- The NIR DIBs at $\lambda_{\text {rest }} 16226.7,16567.0,16588.0$, and $17802.7 \AA$ reported by Geballe et al. (2011) are confirmed only in the spectrum of 4 U $1907+09$.

- The shallow/broad bands at 15225, 15990, 17758, and $17930 \AA$ A reported by Geballe et al. (2011) are not detected. The 15990,17758 , and $17930 \AA$ DIBs are situated in regions with significant telluric line residuals in the X-Shooter spectra presented here. Particularly, the 15225,15990 , and $17758 \AA$ DIBs are among the weakest reported by Geballe et al. (2011), and scaled to the extinction of $\sim 10$ visual magnitudes towards $4 \mathrm{U} 1907+09$, would have depths of only 1.5 , 0.5 , and $1.5 \%$, respectively (to be compared to the weak, but narrow, tentative NIR DIBs detected at a level of $2.0 \%$ in this line-of-sight).

In addition to these previously reported NIR DIBs we report seven new NIR DIB candidates in the spectra of the three 
N. L. J. Cox et al.: Near-infrared diffuse interstellar bands
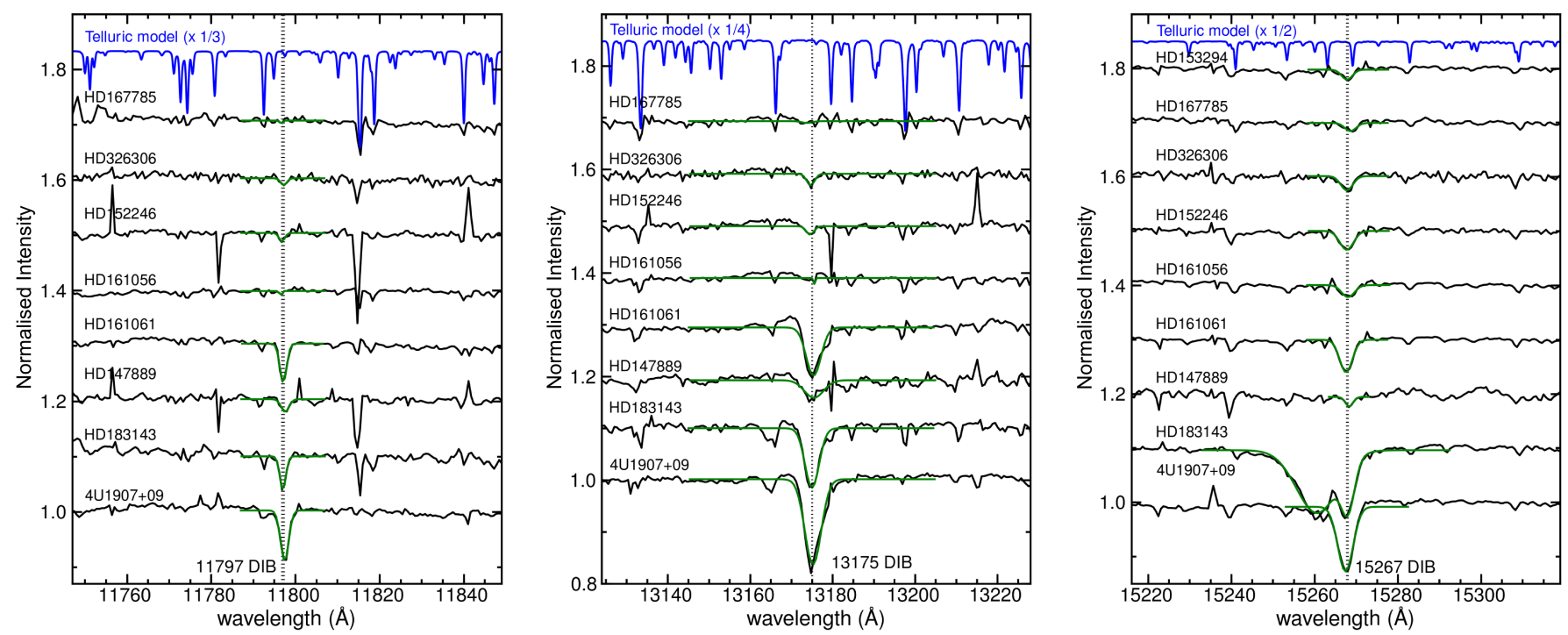

Fig. 4. NIR DIBs at $11797 \AA$ (left panel), $13175 \AA$ (middle panel; Joblin et al. 1990), and $15268 \AA$ (right panel; Geballe et al. 2011) are shown for the X-Shooter sample of sightlines. The spectra have been corrected for the presence of telluric lines and subsequently for the radial velocity of the ISM. The dashed lines indicate the rest wavelength for the respective DIBs. Solid green curves show the Gaussian model fits and the blue solid curve displays the telluric model spectrum.
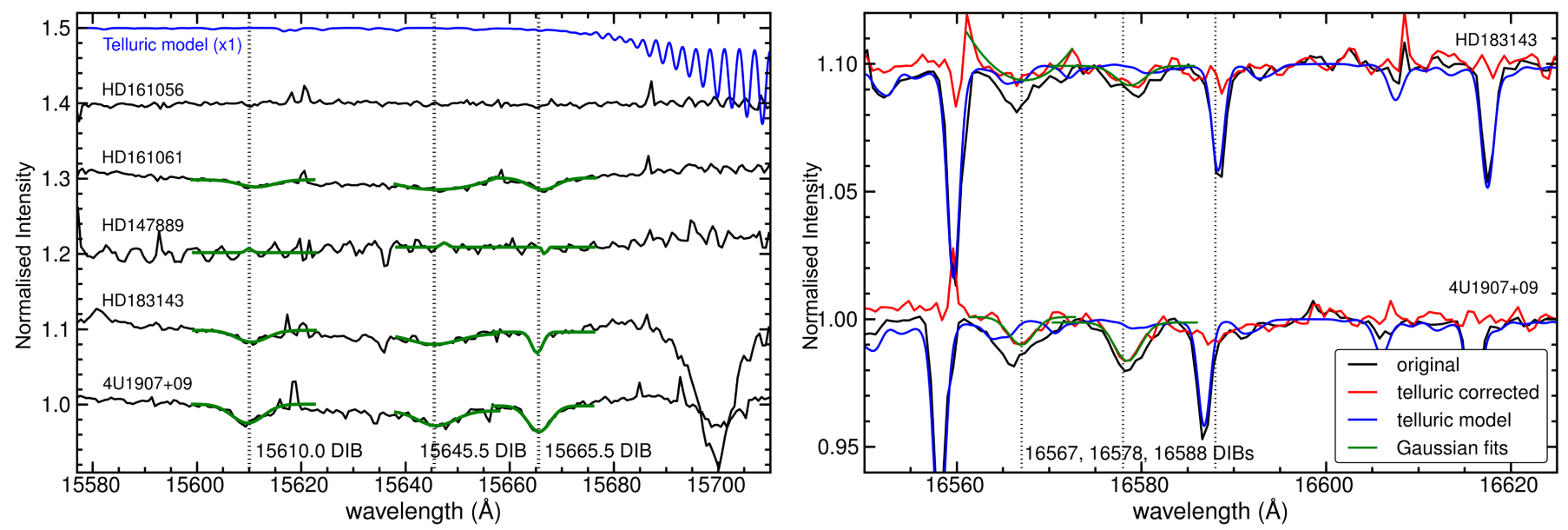

Fig. 5. Same as Fig. 4 but for the 15610, 15646, and $15666 \AA$ NIR DIBs towards five reddened targets (left panel) and for the 16567, 16578, and $16588 \AA ̊ \AA$ NIR DIBs in two reddened lines of sight (right panel). Solid green curves show the Gaussian model fits and the blue solid curve displays the telluric model spectrum. In the right panel the original (normalised) spectra are shown in black while the telluric corrected spectrum is overplotted in red.

most reddened stars (i.e. HD 161061, HD 18314, 4U 1907+09). Similar to the known NIR DIBs these new candidates are not detected towards HD 147889 (Sect. 6). Candidates are listed in Table 2 (bottom section) and respective spectra are shown in Fig. 6. These seven DIB candidates have similar strengths per unit reddening as the known NIR DIBs and their rest wavelengths coincide in different sightlines. Even though the latter criterion provides a strong argument in favour of an interstellar origin, we remain cautious in view of possible limiting factors such as (i) insufficient signal-to-noise ratio for spectra with low reddening, $E_{(B-V)}<1.0 \mathrm{mag}$; (ii) imperfect removal of telluric lines; (iii) confusion with stellar lines, and (iv) the small number of available sightlines with moderate/high-resolution NIR spectra of appropriate interstellar sightlines. Additional possible interstellar features at $12336 \AA$, $20428 \AA, 21387 \AA$, and $21843 \AA$ are present in the spectrum of $4 \mathrm{U} 1907+09$, but not, or only marginally, in spectra of HD 183143 and HD 161061. These lines are also not seen in the spectrum of the non-reddened standard star, so that a stellar origin can be excluded. For completeness these tentative interstellar features are shown in Fig. 7. Further studies at high continuum sensitivity for a large sample are needed to provide further constraints on the presence of DIBs in the NIR range from $1 \mu \mathrm{m}$ to $3 \mu \mathrm{m}$, although in practice results will be limited by how accurate both telluric and stellar atmosphere lines can be accounted for.

\subsubsection{Measurements of NIR DIBs}

We fitted a Gaussian to the NIR DIBs and determined the equivalent width, FWHM and rest-frame wavelength from the best-fit 

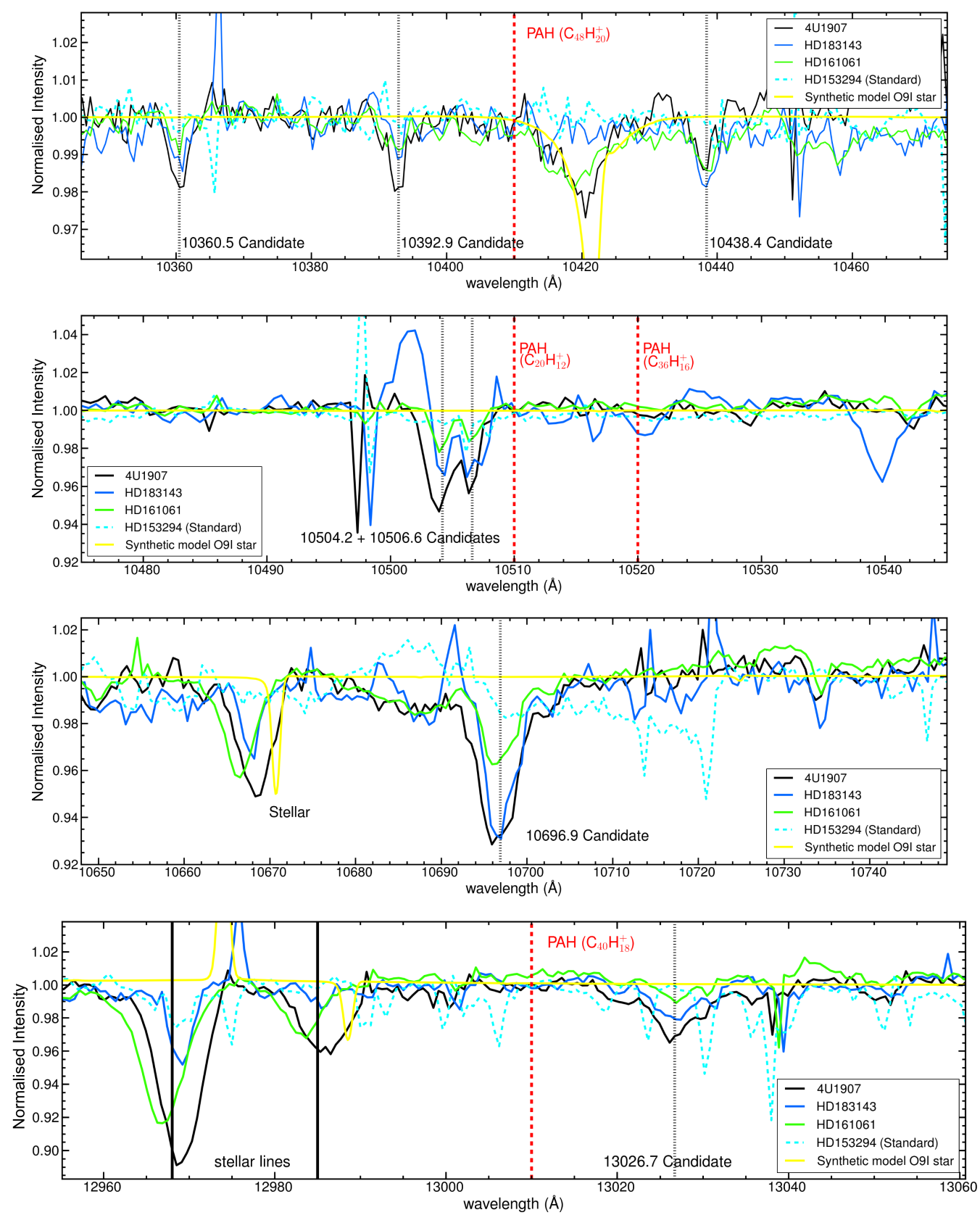

Fig. 6. Candidate NIR DIBs at $\lambda \lambda 10360,10392,10438,10503,10506,10696$, and 13026 A for reddened sightlines towards HD 161061 (green), HD 183143 (blue), and 4U 1907+09 (black). The spectra have been shifted to the interstellar rest frame. The dotted black lines indicate the rest wavelength for the candidate NIR DIBs. Solid black lines in the bottom panel indicate the position of stellar lines. The dashed red lines correspond to the laboratory positions of main absorption bands of several mid-sized PAHs reported by Mattioda et al. (2005). The peaks of the stellar lines (at $10422,10670,10830,12969$, and $12984 \AA$ ) do not coincide as a result of differences in radial velocity of the stars. The telluric standard spectrum of HD 153294 (dashed cyan) and stellar atmosphere model of an O9Ia star (solid yellow) are overplotted for reference.

parameters. Equivalent widths were also measured by numerical integration, yielding similar results within the uncertainties. The results for the detected NIR DIBs in each sightline are given in Table 2.

\section{NIR DIB properties}

Given the number of NIR DIB detections, we can now compare some of their properties in more detail to those of the optical DIBs. We will in particular look at their widths, strengths and correlations.

\subsection{Rest wavelengths and DIB profiles}

The moderate resolving power $(R \sim 10000)$ of X-Shooter is insufficient to resolve smaller substructure or weak asymmetries in the NIR DIB absorption line profiles. However, compared to the work by Geballe et al. (2011), our lines of sight suffer much less from line broadening due to blending of multiple velocity components in the line of sight that are Doppler shifted as a result of Galactic rotation. With the exception of 4U 1907, Doppler broadening is not expected to exceed $10 \mathrm{~km} \mathrm{~s}^{-1}$ (or $0.5 \AA$ at $15000 \AA$ ); thus, the observed FWHM from $1.9 \AA$ to $7.0 \AA$ (see 

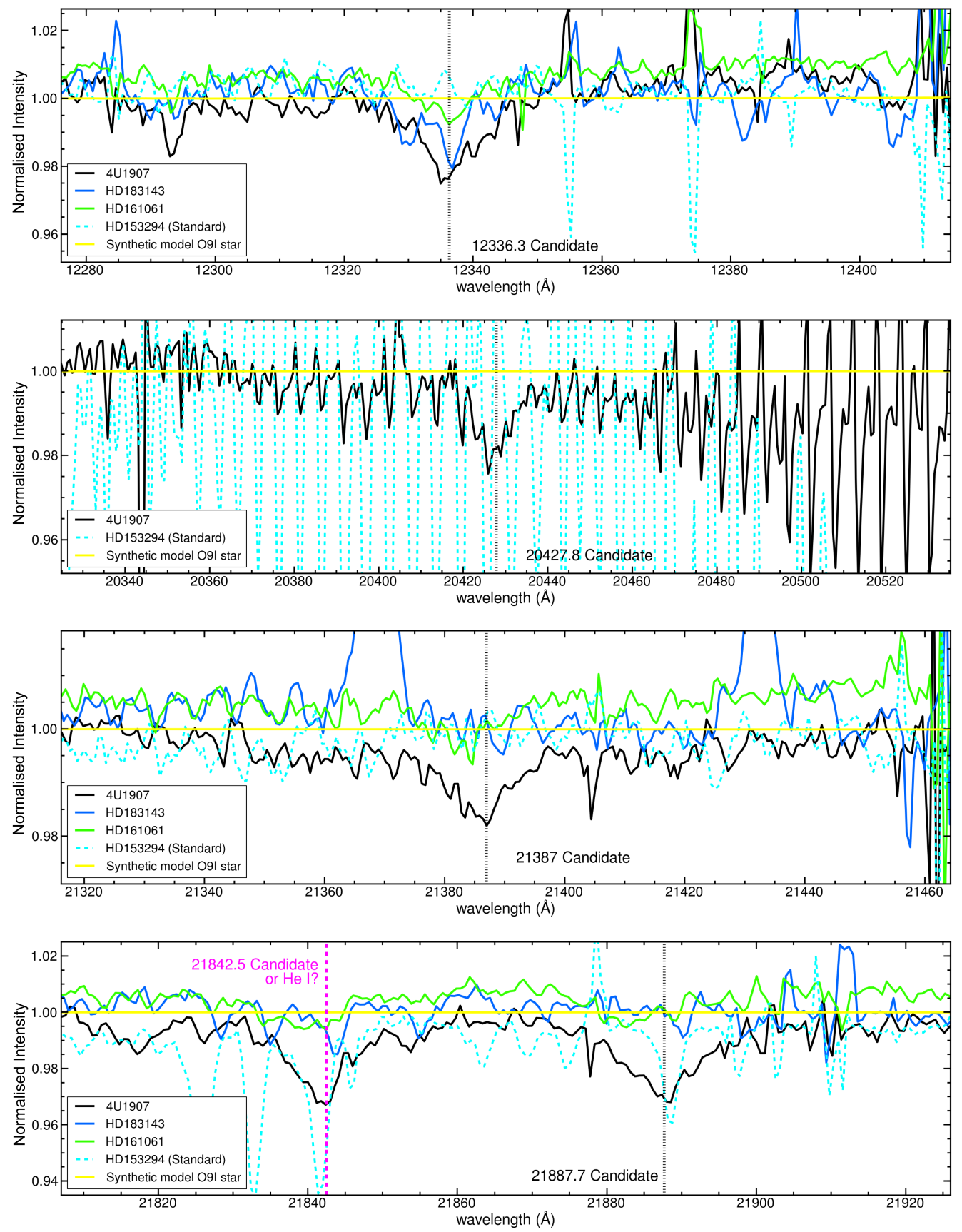

Fig. 7. Tentative NIR DIB candidates at $12336.3 \AA$, $20427.8 \AA$, $21387.0 \AA$, $21842.5 \AA$, and $21887.7 \AA$ (from top to bottom) towards the reddened star 4U 1907 (and HD 183143 and HD 161061 where applicable). Dotted vertical lines indicate the position of the tentative NIR DIBs. The telluric standard spectrum of HD 153294 (dashed cyan) and stellar atmosphere model of an O9Ia star (solid yellow) are overplotted for reference. The feature at $21842.5 \AA$ is possibly (a blend with) a stellar He I line.

Table 3) correspond to intrinsic FHWM ${ }^{2}$ from $1.4 \AA\left(20 \mathrm{~km} \mathrm{~s}^{-1}\right)$ to $6.8 \AA\left(77 \mathrm{~km} \mathrm{~s}^{-1}\right)$.

Owing to this lack of Galactic rotation Doppler shift line broadening and the precise measurement of the line-of-sight radial velocity (see 3.1), we can derive accurate rest wavelengths (reported in air) for the NIR DIBs listed in Table 2. The significant Doppler broadening towards 4U 1907 has been discussed in Cox et al. (2005).

The measured widths (FWHM) of the NIR DIBs are listed in Table 3. The NIR DIBs at $15268,15665,16567$, and $16578 \AA$

$2 F H W M_{\text {intrinsic }}=\sqrt{F H W M_{\text {observed }}^{2}-F H W M_{\text {instrumental }}^{2}}$. are narrow and have widths comparable to the earlier reported NIR DIBs at 9577, 9632, 11797 , and $13175 \AA$. We find that the widths of the 15268 and $15665 \AA$ DIBs are smaller than those reported by Geballe et al. (2011), suggesting that Doppler velocity broadening affects their measurements. This is not the case for the broad 15610 and $17800 \AA$ NIR DIBs whose widths are similar to those by Geballe et al. (2011). For the three reddened lines of sight with a narrow interstellar velocity structure we furthermore find that $F W H M(11797)=1.9 \pm 0.4 \AA$ (somewhat narrower than the $2.7 \pm 0.3 \AA$ reported by Joblin et al. 1990) and $F W H M(13175)=4.5 \pm 1.2 \AA$ (comparable to the $4.0 \pm 0.5 \AA$ measured by Joblin et al. 1990). 
Table 3. FWHM (in $\AA$; uncertainties in parentheses) of NIR DIBs in reddened sightlines observed with $\mathrm{X}$-Shooter.

\begin{tabular}{lllll}
\hline \hline \multirow{2}{*}{$\lambda_{\mathrm{DIB}}{ }^{a}$} & \multicolumn{5}{c}{$F W H M(\AA)$} \\
& HD 161061 & HD 147889 & HD 183143 & $4 \mathrm{U} 1907$ \\
\hline 9577 & $3.9(0.12)$ & - & $3.3(0.07)$ & $4.8(0.07)$ \\
9632 & $2.7(0.05)$ & - & $2.5(0.03)$ & $3.6(0.06)$ \\
10780 & $1.7(0.1)$ & - & $1.8(0.1)$ & $2.4(0.2)$ \\
11797 & $1.9(0.1)$ & $2.2(0.7)$ & $1.7(0.1)$ & $2.3(0.1)$ \\
13175 & $4.2(0.3)$ & $5.5(1.5)$ & $4.0(0.3)$ & $4.7(0.2)$ \\
15268 & $3.6(0.3)$ & $2.2(0.4)$ & $3.7(0.3)$ & $4.4(0.3)$ \\
15610 & $7.8(3.0)$ & - & $7.0(2.6)$ & $7.1(2.5)$ \\
15646 & - & - & $11.0(5.8)$ & $8.3(2.7)$ \\
15666 & $6.3(1.5)$ & - & $2.5(0.3)$ & $5.1(0.4)$ \\
16227 & - & - & - & $33(7)$ \\
16567 & - & - & - & $3.2(0.5)$ \\
16578 & - & - & $3.7(1.2)$ & $3.3(0.5)$ \\
16588 & - & - & - & $2.9(0.9)$ \\
17803 & - & - & - & $13(4)$ \\
& & & & \\
\hline 10361 & $2.0(0.5)$ & - & $3.3(2.3)$ & $3.1(0.5)$ \\
10393 & $3.2(1.0)$ & - & $1.4(0.4)$ & $2.8(0.5)$ \\
10438 & $2.6(0.4)$ & - & $3.5(0.5)$ & $4.0(0.9)$ \\
$10504^{b}$ & 1.4 & - & 1.2 & 2.3 \\
$10507^{b}$ & 1.5 & - & 4.8 & 1.5 \\
10697 & $3.8(0.6)$ & - & $3.9(0.5)$ & $4.9(0.3)$ \\
$13027^{b}$ & 4.7 & - & 5.5 & 5.7 \\
\hline
\end{tabular}

Notes. ${ }^{(a)}$ Measured rest peak wavelengths in air are given in Table 2. (b) Complex profile; possible blended lines or telluric residuals. No sensible uncertainties could be determined for (individual) components.

Table 4. Equivalent widths (uncertainties in parentheses) for the 11797 , 13175 , and $15268 \AA$ A NIR DIBs reported previously in the literature.

\begin{tabular}{|c|c|c|c|c|}
\hline \multirow[t]{2}{*}{ Target } & \multirow{2}{*}{$\begin{array}{l}E_{(B-V)} \\
(\mathrm{mag})\end{array}$} & \multicolumn{3}{|c|}{ Equivalent widths (m̊) } \\
\hline & & 11797 & 13175 & 15268 \\
\hline HD $24398^{b}$ & 0.34 & $32(9)$ & $50(20)$ & - \\
\hline $\mathrm{HD} 223385^{b}$ & 0.59 & $280(38)$ & $460(34)$ & - \\
\hline HD $20041^{b}$ & 0.73 & $34(4)$ & $262(35)$ & - \\
\hline HD $183143^{b}$ & 1.04 & $169(29)$ & $451(37)$ & - \\
\hline HD $194279^{b}$ & 1.22 & $336(54)$ & $560(55)$ & - \\
\hline $\mathrm{BD}+404223^{a}$ & 1.93 & - & $860(60)$ & - \\
\hline $\mathrm{BD}+404220^{b}$ & 2.00 & $394(44)$ & $870(60)$ & - \\
\hline $\mathrm{GCS} 3-2^{a}$ & $\sim 6.5$ & - & - & $1500(100)$ \\
\hline $\mathrm{gF} 362^{a}$ & $7.0(0.6)$ & - & $3130(100)$ & - \\
\hline
\end{tabular}

Notes. ${ }^{(a)}$ Equivalent width measurements from Geballe et al. (2011). Visual extinction for GCS3-2, qF362, and BD+40 4223 is $~ 20$, $21.7 \pm 1.7$, and $5.97 \mathrm{mag}$, respectively. ${ }^{(b)}$ Equivalent width measurements from Joblin et al. (1990). HD 183143 is the only X-Shooter line-of-sight also observed by Joblin et al. The equivalent width of the $11797 \AA$ DIB observed with X-Shooter is $40 \%$ smaller, while those for the $13175 \AA$ DIB agree within the uncertainties.

Finally, it is interesting that the widths of the 11797 and $13175 \AA$ NIR DIBs in the single cloud line-of-sight towards HD 147889 are similar to those towards the multicloud object 4U 1907. However, the NIR DIBs are weak in this sightline, and consequently uncertainties are large.

\subsection{DIB strengths}

Table 2 lists the equivalent widths for the NIR DIBs in the highly reddened lines of sight. It is clear that the detected NIR DIBs are fairly strong. The average NIR DIB strength per unit reddening, $W(\mathrm{DIB}) / E_{(B-V)}$ ranges from $0.07 \AA \mathrm{mag}^{-1}$ to $0.35 \AA \mathrm{mag}^{-1}$ in our lines of sight while the total NIR DIB equivalent width per unit reddening (for the five strongest NIR DIBs) ranges from $\sim 0.6 \AA \mathrm{mag}^{-1}$ to $1.4 \AA \mathrm{mag}^{-1}$. This is similar to the total strength of the seven strongest optical DIBs, although the wavelength dependence in the equivalent width measurement favours the NIR DIBs equivalent width (in wavelength units) by a factor of two to three.

For an effective wavelength of $1.5 \mu \mathrm{m}$ and assuming optically thin line absorption, we thus find that $f \times N_{\mathrm{NIRDIB}}=$ $3-7 \times 10^{11} \mathrm{~cm}^{-2}$ for all NIR DIBs combined; $f$ is then an effective oscillator strength. For the strongest optical DIBs $\left(\lambda_{\text {effective }}=\right.$ $6000 \AA), f \times N_{\text {DIB }}=5 \times 10^{12} \mathrm{~cm}^{-2}$. Thus, if oscillator strengths are similar for both optical and NIR DIBs (as is e.g. the case for molecule No. 25 discussed in Sect. 5), the abundance of NIR DIB carriers is an order of magnitude lower than that of the carriers of the strongest optical DIBs. If, however, the optical and NIR DIBs arise from the same species in the same state, their respective column densities should be equal and hence the oscillator strengths of the NIR DIBs are an order of magnitude smaller. Note however that such a situation is not likely, given the imperfect correlation between NIR and optical DIBs (see below).

\subsection{DIB strength correlations}

In an effort to understand the relation between the NIR DIB carriers and those of the optical DIBs, we will look at correlations between DIB strengths and reddening, and mutual correlations between the NIR DIBs and optical DIBs. This can only be done in a meaningful way with a large enough sample of sightlines; we therefore only include the $\lambda \lambda 11797,13175$, and 15268 NIR DIBs for which we have at least six measurements; there are too few data points for the other NIR DIBs.

\subsubsection{Correlation with reddening}

The correlation coefficients ${ }^{3} r$ between the DIB strengths and reddening range from 0.80 (for the 6203 and $6270 \AA$ DIBs) to 0.95 (for the $5797 \AA$ DIB) for the optical DIBs in our sample confirming the well established positive but not perfect correlation between $W$ and $E_{(B-V)}$ (e.g. Herbig 1993; Cami et al. 1997; Friedman et al. 2011).

Figure 8 shows the measured equivalent widths for the $\lambda \lambda 11797,13175$, and 15268 NIR DIBs as a function of reddening, and linear regression fits to the data. For the linear regression the $95 \%$ confidence interval is also indicated (shaded area). The corresponding correlation and linear fit coefficients are given in Table 5. Notacibly, the strength of the $11797 \AA$ NIR DIB falls well above the linear trend for three lines of sight reported in Joblin et al. (1990). At this time we cannot confirm if any systematics (such as telluric residuals) affect these measurements or if they are truly peculiar, even though for the only sightline in common, HD 183143, we report a $\sim 35 \%$ smaller equivalent width. Although the sample is limited, we find for most NIR DIBs a clear, but imperfect, trend of increasing DIB strength with increasing $E_{(B-V)}$.

3 We always imply Pearson linear correlation coefficients in this work. 

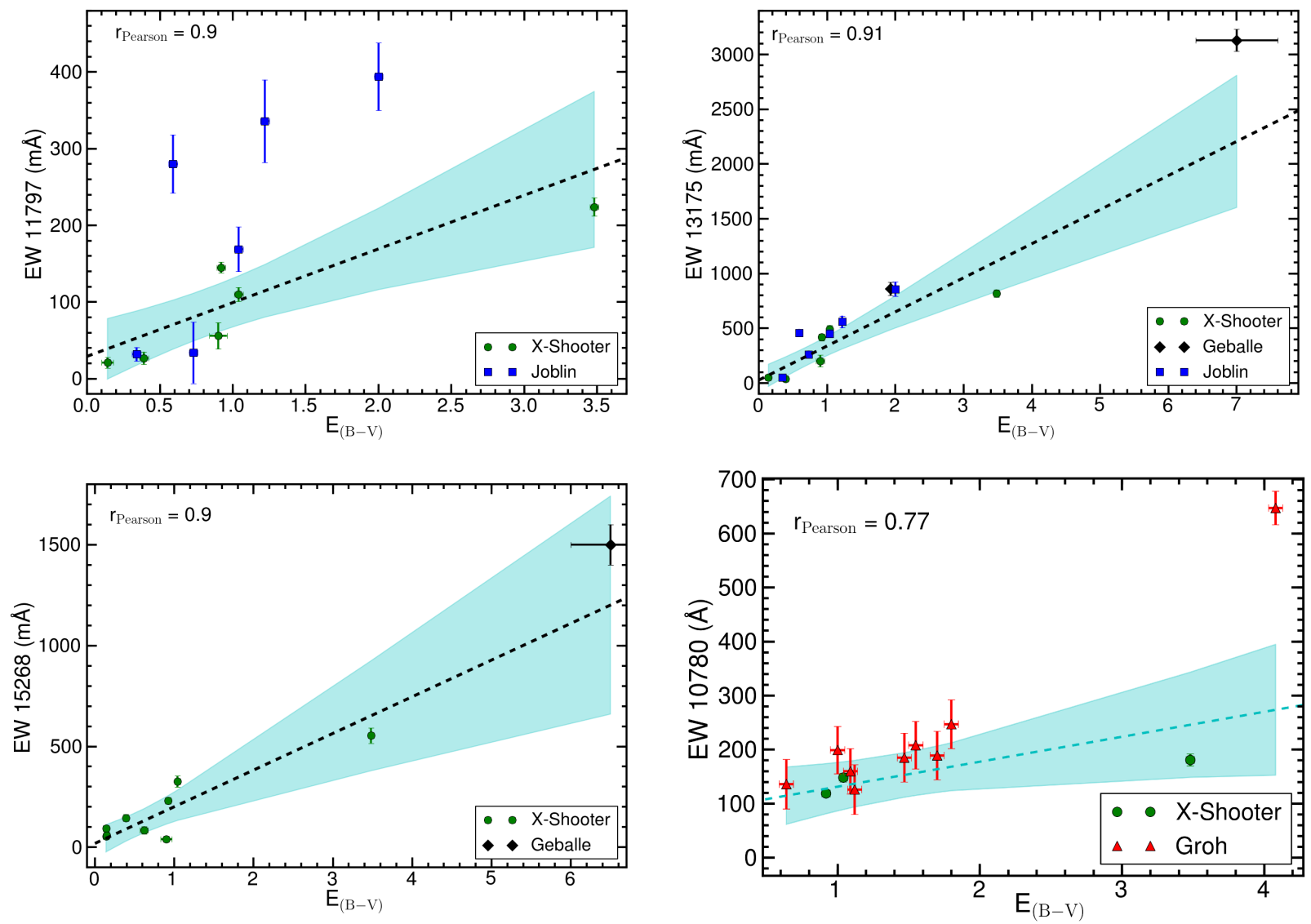

Fig. 8. $\lambda \lambda 11797,13175,15268$, and 10780 NIR DIB equivalent widths $(\AA)$ as a function of reddening, $E_{(B-V)}$ including measurements from Joblin et al. (1990, blue squares), Geballe et al. (2011, see also Table 2; black diamonds), Groh et al. (2007, red triangles) and this work (green circles). The linear least-square fit is indicated by the black dashed line (parameters are given in Table 5) and the shaded cyan area indicates the $95 \%$ confidence interval.

\subsubsection{DIB families}

Table 5 also lists the correlation coefficients between the three selected NIR DIBs and the optical DIBs. For consistency and to avoid systematics these correlation coefficient only take the $\mathrm{X}$-Shooter data into account.

Correlation coefficients are $r>0.8$ between the $15268 \AA$ NIR DIB and the optical DIBs. The optical DIBs we consider are also well correlated with each other, $r \geq 0.9$. All correlations between the $15268 \AA$ DIB and the optical DIBs are shown in Fig. 10. The $15268 \AA$ NIR DIB is only moderately correlated $(r \sim 0.9)$ with both the $11797 \AA$ or $13175 \AA$ NIR DIBs. The latter two are strongly correlated with each other, $r=0.97$.

Since the number of data points is small (between six and nine) and there is significant scatter on the derived linear regressions we caution against over-interpretation of the correlation coefficients presented here. A larger sample is required for each diffuse band to substantiate or invalidate these preliminary relations, which could be nevertheless useful in, for example, preparing follow-up observations. Nonetheless, our measurements reveal that optical and NIR DIBs display a similar behaviour with respect to interstellar physical conditions as probed by different lines of sight. Furthermore, the data tentatively suggest that the NIR DIB at $15268 \AA$ NIR DIB is moderately well correlated with all the optical DIBs. The weakest correlation is found for the optical DIBs at 5797 and $5849 \AA$. The relation between NIR DIBs and the $5780 \AA$ DIB (which is considered as a good tracer of neutral hydrogen; Herbig 1993) is discussed in Sect. 6. Considering the small sample size we do not consider further subdivision of the sample according to, for example, $\sigma / \zeta$-type, etcetera.

\section{NIR DIBs and large PAH cation bands}

In the context of the DIB-PAH proposal, the NIR range could be particularly interesting since many PAH ions have strong electronic transitions in this wavelength range (Mattioda et al. 2005). Specifically, these authors measured the spectra of $27 \mathrm{PAH}$ cations and anions, ranging in size from $\mathrm{C}_{14} \mathrm{H}_{10}$ to $\mathrm{C}_{50} \mathrm{H}_{22}$, and found that they have strong and broad absorption bands between 0.7 and $2.5 \mu \mathrm{m}$. Smaller PAHs on the other hand have their strongest transitions in the optical or UV. Since it is thought that the population of interstellar PAHs is dominated by species in the $N_{\mathrm{C}}=50$ range (see e.g. Tielens 2008), the NIR is ideally suited to study and possibly identify these species in space.

The PAH ion absorption spectra presented by Mattioda et al. (2005) are typically characterised by one or two strong transitions accompanied by several weaker transitions. Ten of the PAH cations presented have bands with oscillator strengths $f \leq$ 0.02 longwards of $1.0 \mu \mathrm{m}$. Here, we make an initial comparison between these laboratory measurements and the NIR DIBs. Note however that the absorption band wavelengths reported by Mattioda et al. (2005) result from matrix-isolation experiments and hence suffer from an unknown shift in position (by as much as tens of Angstrom) as well as from band broadening due to 

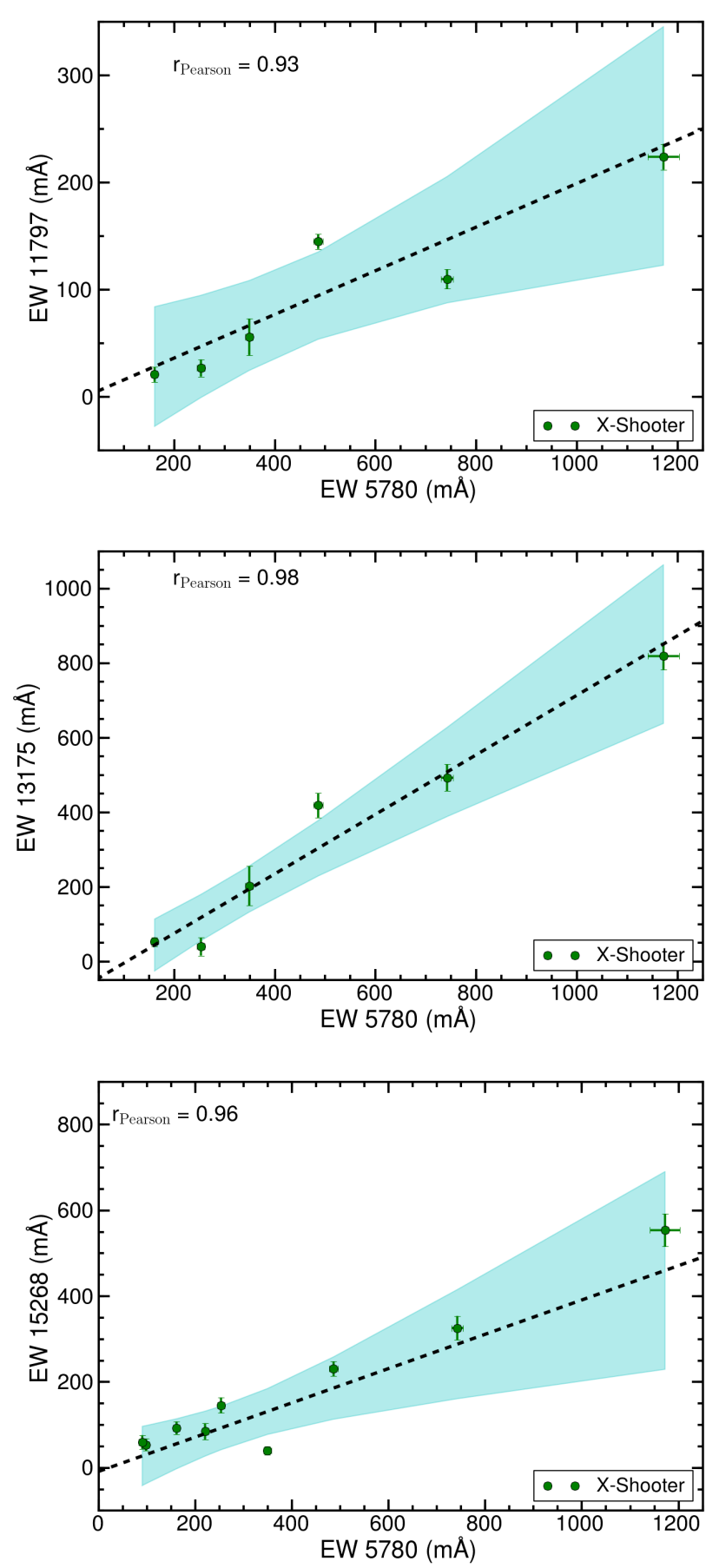

Fig. 9. Mutual correlation between the $5780 \AA$ DIB and the $\lambda \lambda 11797$, 13175 , and 15268 NIR DIBs. The correlation coefficients are given in each panel. The linear least-square fit is indicated by the black dashed line (parameters are given in Table 5) and the shaded cyan area indicates the $95 \%$ confidence interval.

interaction with the solid lattice. Thus, while a direct identification of PAH cations is not possible with this data set, a comparison between the laboratory data and the NIR DIBs can offer some insights into this population of carbonaceous species. Here, we present some of the more interesting cases; the numbering refers to that used by Mattioda et al. (2005):

1. Molecule No. $10\left(\mathrm{C}_{20} \mathrm{H}_{12}^{+}\right)$has two strong bands at $10510 \AA$ $(f=0.077)$ and $9277 \AA(f=0.008)$. We identified two
Table 5. Correlation coefficients $r$ and best-fit parameters of the linear regression $y=a * x+b$ for the listed parameter pairs for the X-Shooter data.

\begin{tabular}{llll}
\hline \hline Parameter pair $y-x^{a}$ & $r$ & $a^{b}$ & $b(\mathrm{m \AA})^{b}$ \\
\hline$W(11797)-E_{(B-V)}$ & 0.90 & $70(17)$ & $29(19)$ \\
$W(13175)-E_{(B-V)}$ & 0.91 & $311(44)$ & $28(50)$ \\
$W(10780)-E_{(B-V)}$ & 0.77 & $46(19)$ & $85(33)$ \\
$W(15268)-E_{(B-V)}$ & 0.90 & $182(40)$ & $19(33)$ \\
$W(13175)-W(11797)$ & 0.97 & $3.76(0.42)$ & $37(43)$ \\
$W(11797)-W(5780)$ & 0.92 & $0.20(0.55)$ & $-4(29)$ \\
$W(13175)-W(5780)$ & 0.98 & $0.80(0.91)$ & $-84(37)$ \\
$W(15268)-W(5780)$ & 0.96 & $0.40(0.11)$ & $-8(37)$ \\
$W(15268)-W(5797)$ & 0.91 & $0.87(0.35)$ & $14(44)$ \\
$W(15268)-W(5849)$ & 0.83 & $1.40(1.02)$ & $43(56)$ \\
$W(15268)-W(6196)$ & 0.95 & $3.20(0.96)$ & $-4(36)$ \\
$W(15268)-W(6203)$ & 0.97 & $1.32(0.24)$ & $4(24)$ \\
$W(15268)-W(6270)$ & 0.97 & $1.53(0.26)$ & $24(21)$ \\
$W(15268)-W(6613)$ & 0.95 & $0.74(0.22)$ & $-2(39)$ \\
$W(15268)-W(11797)$ & 0.90 & $1.98(0.56)$ & $31(54)$ \\
$W(15268)-W(13175)$ & 0.93 & $0.57(0.13)$ & $48(39)$ \\
\hline
\end{tabular}

Notes. Equivalent widths, $W$, given in $\mathrm{m} \AA .{ }^{(a)}$ Equivalent width of the NIR DIB at the given wavelength or interstellar reddening. ${ }^{(b)}$ The linear regression coefficients including uncertainties as shown in Figs. 8-10.

new candidate DIBs at $10504 \AA$ and $10506 \AA$, but could not verify the presence of the second, weaker band near $9277 \AA$ due to a strong telluric line forest in this range.

2. Molecule No. $16\left(\mathrm{C}_{36} \mathrm{H}_{16}^{+}\right)$shows a strong laboratory band at $10520 \AA(f=0.031)$, with two weaker bands (by one order of magnitude) at $10220 \AA$ and $9196 \AA$. Again, two tentative NIR DIBs are identified at $10504 \AA$ and $10506 \AA$. No feature is revealed at $10220 \AA$, but a shallow broad feature is suspected at $9212 \AA$.

3. Molecule No. $18\left(\mathrm{C}_{40} \mathrm{H}_{18}^{+}\right)$has a strong band at $10410 \AA(f=$ $0.024)$, near the new candidate at $10438 \AA$. The weaker bands at $9789 \AA$ and $8918 \AA$ (laboratory) could not be discerned in the X-Shooter spectra.

4. Molecule No. $19\left(\mathrm{C}_{40} \mathrm{H}_{18}^{+}\right)$has a strong band at $13010 \AA$ ( $f=$ 0.026), near the new candidate at $13026 \AA$. Secondary laboratory bands with $f \sim 0.002$ are present at 9233, 7854, and $7693 \AA$. As noted above, a NIR band may be present at $9212 \AA$. Furthermore, strong DIBs have been identified at 7686.5, 7705.9, and $7710 \AA$ (Jenniskens \& Désert 1994).

5. Molecule No. $22\left(\mathrm{C}_{42} \mathrm{H}_{22}^{+}\right)$has a strong band at $10790 \AA(f=$ $0.042)$, near the new candidate at $10780 \AA$. The secondary bands at 9386 and $7209 \AA(f \sim 0.004)$ are not found.

6. Molecule No. $25\left(\mathrm{C}_{48} \mathrm{H}_{20}^{+}\right)$has a strong band at $10410 \AA(f=$ 0.089), near the new candidates at $10392 \AA$ and $10438 \AA$. Its strongest feature is at $6941 \AA(f=0.160)$ which is near a strong broad DIB at $6939 \AA$ with FWHM of $21.3 \AA$.

We do not find any features in the vicinity of the main transitions reported for molecules No. $23\left(\mathrm{C}_{44} \mathrm{H}_{20}^{+} ; f=0.025\right)$, No. 24 $\left(\mathrm{C}_{48} \mathrm{H}_{20}^{+} ; f=0.061\right)$, No. $26\left(\mathrm{C}_{48} \mathrm{H}_{22}^{+} ; f=0.031\right)$.

The above comparison shows several coincidences between the newly detected NIR DIBs and the laboratory spectra of PAH cations, thus illustrating the potential of this low-congested 
N. L. J. Cox et al.: Near-infrared diffuse interstellar bands
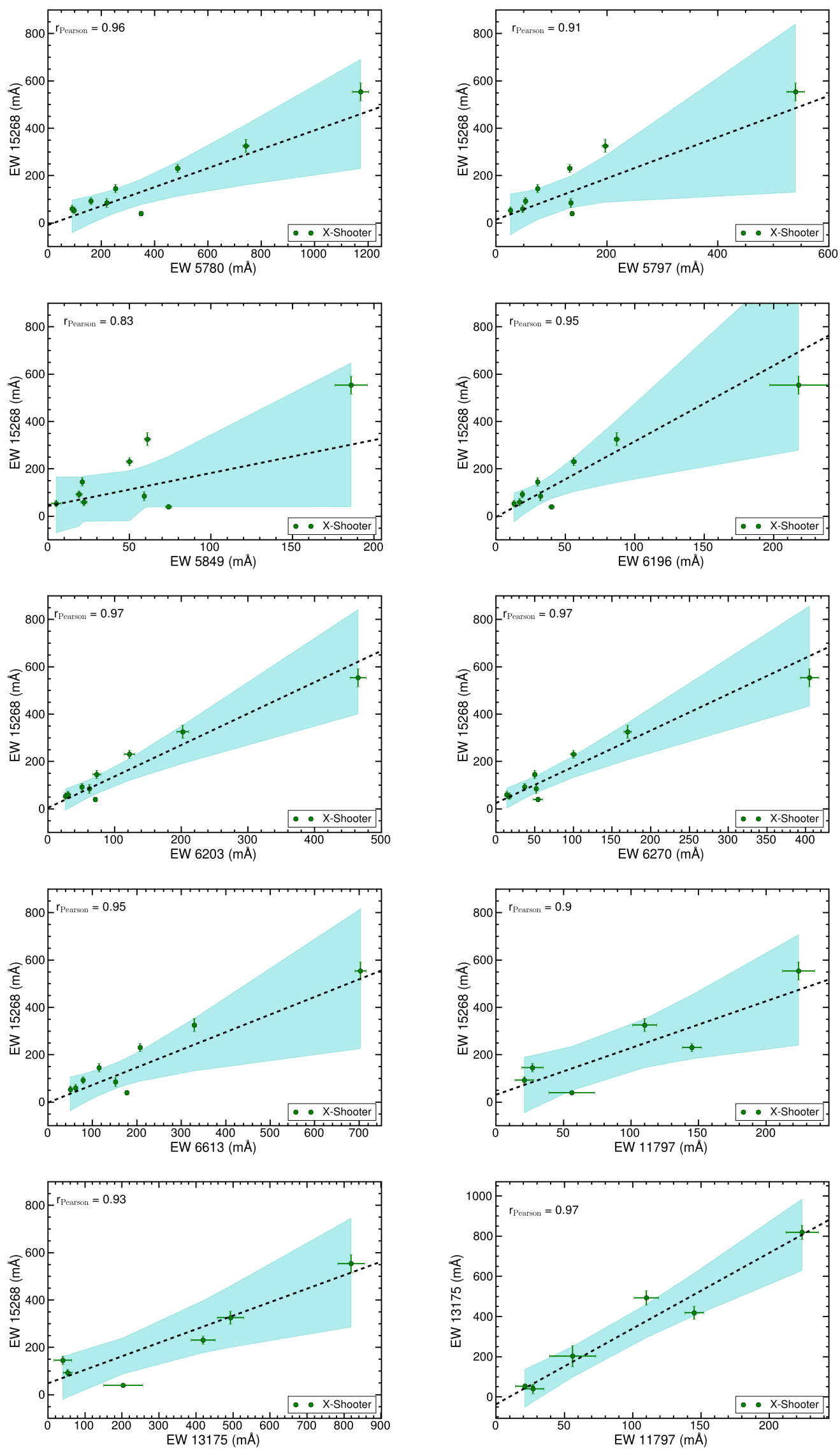

Fig. 10. Relation between the $15268 \AA$ A NIR DIB and the strong optical DIBs, and the 11797 and $13175 \AA$ NIR DIBs. The correlation coefficients are given in each panel. In addition the 11797-13 175 NIR DIBs are compared in the bottom right panel. The linear least-square fit is indicated by the black dashed line (parameters are given in Table 5) and the shaded cyan area indicate the 95\% confidence interval. 
range of the DIB spectrum. However, the measured PAH cation bands are much broader (at least tens of $\AA$ ) than the rather narrow NIR DIBs. While part of the measured bandwidth for the PAHs certainly results from matrix broadening effects, it is clear that PAH cation bands are intrinsically broad as well, and thus possibly too broad to match the NIR DIBs. A conclusive answer can therefore only come from precise transition wavelengths and information on the intrinsic band profiles and widths, obtained from gas-phase spectra of possible carrier molecules as has been illustrated for DIBs in the optical range (see e.g. Gredel et al. 2011; Salama et al. 2011).

\section{The translucent cloud toward HD 147889}

In Sect. 3.2 we found that many of the NIR DIBs towards HD 147889 are weak compared to sightlines with similar reddening. On the other hand, strengths of the optical DIBs are consistent with those found in similar lines of sight. For instance the optical DIBs toward HD 161061, HD 147889 and HD 183143 have similar strengths per unit reddening, while the NIR DIBs at 9577, 9632, and $15628 \AA$ are weaker. The strengths of the 11797 and $13175 \AA$ NIR DIBs toward HD 147889 are in line with the good linear relation with the $5780 \AA$ A DIB strength, while the $15268 \AA$ NIR DIB is unusally weak compared to the general trend (Fig. 9).

The line of sight toward HD 147889 sightline passes through a well known and extensively studied translucent cloud with a visual extinction of $\sim 4$ mag (Ruiterkamp et al. 2005). Its mean hydrogen density and molecular hydrogen fraction are high, $n_{\mathrm{H}}=1200 \pm 500 \mathrm{~cm}^{-3}$ and $f_{\mathrm{H}_{2}}=0.4$. The effective local interstellar radiation field strength for this cloud is 11 times as strong as the Galactic average. Ruiterkamp et al. (2005) computed the ionisation balance of a set of small to intermediate size PAHs to construct a PAH absorption spectrum ranging from the UV to the NIR. These models showed a non-negligible fraction of anions and a significant fraction of cations. The prediction for this lineof-sight was that if DIBs are due to compact and non-compact PAHs homologue series (with $10<\mathrm{C}$-atoms $<50$ ) then more DIB features should be present longwards of $10000 \AA$. Thus, the absence of NIR DIBs could indicate that for HD 147889 large non-compact PAHs are absent or altered, for example due to substantial hydrogenation, resulting in weak bands. In addition, the ionisation fraction in this translucent cloud is less than $30 \%$ for small PAHs with less than 30 carbon atoms, while the anion fraction is over $20 \%$. Thus the absence of the NIR DIBs (and $5849 \AA$ DIB) could be related to the low ionisation fraction of smaller PAHs in this line-of-sight as compared to that in diffuse clouds. A lower ionisation fraction - basically due to self-shielding is expected for translucent clouds with a high molecular abundance (Snow \& McCall 2006). Hence, also the reduced strength of the $15268 \AA$ NIR DIB with respect to the $5780 \AA$ DIB which is known to trace particularly well the amount of neutral hydrogen (Herbig 1995; Friedman et al. 2011). Due to the low ionisation potential of large ( $>100 \mathrm{C}$-atoms) PAHs, these would have a large ionisation fraction $(\geq 50 \%)$ even in this translucent cloud.

\section{Summary and conclusions}

This paper presents moderate-resolution optical and NIR spectra, using X-Shooter at the VLT, for eight Galactic early-type stars with interstellar reddening values $\left(E_{B-V}\right)$ ranging from 0 to $3.5 \mathrm{mag}$ (or $10 \mathrm{mag}$ visual extinction correspondingly). We present and discuss the presence and properties of NIR DIBs in these sightlines. Specifically, we have obtained the following results for the presence of DIBs:

\section{Detection of NIR DIBs}

- We detect nine of the thirteen new NIR DIBs that were reported by Geballe et al. (2011), and can confirm their interstellar nature from their general correlation with reddening and from the stationary nature of the rest wavelengths when corrected for the radial velocity of the interstellar cloud in the line-of-sight.

- We propose seven new NIR DIB candidates based on their detection in the reddened spectra of HD 183143 and 4U $1907+09$.

Thus, a total of 14 NIR DIBs are confirmed at wavelengths longer than $9500 \AA$; and up to 15 new NIR DIB candidates are reported -11 of which are discussed in this work for the first time.

\section{Properties and behaviour of NIR DIBs}

- Most of the NIR DIBs are intrinsically fairly narrow with $F W H M \sim 2-7 \AA$; only the 16227 and $17803 \AA$ NIR DIBs are broader.

- Although the NIR DIBs are fairly strong, their carrier abundance is about an order of magnitude smaller than the strongest optical DIBs.

- The 11797, 13175 , and $15268 \AA$ NIR DIBs are found to correlate well with the $5780 \AA$ DIB, and by extension correlate better with $H_{I}$ than with $E_{(B-V)}$. The strength ratio of 2 between the 5780 and 15268 DIBs implies that $(N f)_{5780} /(N f)_{15268}=14$; however, the weak 15268 DIB toward HD 147889 precludes a common carrier for the two DIBs.

- Laboratory spectra of large PAH cations show some coincidences with the NIR DIBs; however, their bands are probably to broad to explain the NIR DIBs.

- The NIR DIBs in the translucent cloud towards HD 147889 are nearly absent while most optical DIBs have average strength. Since the ionisation fraction of small to mediumsized PAHs is low in this cloud, this hints at ionised species as the carriers of the NIR DIBs.

The NIR range has proven a fertile wavelength range to search for new DIBs. Given our survey, most strong NIR DIBs between $10000 \AA$ and $25000 \AA$ are likely detected. However, numerous more NIR DIBs could be hiding in the noise, in particular in those regions affected most severely by residuals from telluric line removal procedures. Undoubtedly, further advances in technology, instrumentation and modelling of telluric and stellar atmospheres will lead to the detection of more NIR DIBs.

The NIR DIBs allows one to study the DIBs in highly extincted lines of sight that are inaccessible to optical spectroscopy. This offers some prospects to observe NIR DIBs and PAH infrared emission bands in the same environments. This would most certainly provide a critical test for the PAH-DIB hypothesis. To connect such studies back to optical DIBs, extensive studies are required of both optical and NIR DIBs in a large sample of sightlines, including detailed analyses of their relation to known molecular interstellar species.

Acknowledgements. We thank the referee for a thorough reading of the manuscript and several insightful comments that improved the paper. N.L.J.C. 
N. L. J. Cox et al.: Near-infrared diffuse interstellar bands

thanks the staff at Paranal for their help in optimising the execution of our programme, and X-Shooter instrument scientists at ESO for assistance in data handling issues. J.C. acknowledges support from the Natural Sciences and Engineering Council of Canada (NSERC). We thank Lucas Ellerbroek for guidance in using Spextool to correct the data for telluric absorption lines. This research has made use of the Simbad database operated by CDS in Strasbourg.

\section{References}

Adamson, A. J., Kerr, T. H., Whittet, D. C. B., \& Duley, W. W. 1994, MNRAS, 268,705

Cami, J., Sonnentrucker, P., Ehrenfreund, P., \& Foing, B. H. 1997, A\&A, 326, 822

Cordiner, M. A. 2014, in IAU Symp. 297, eds. J. Cami, \& N. L. J. Cox, 41

Cox, N. L. J. 2011, in EAS Pub. Ser. 46, eds. C. Joblin, \& A. G. G. M. Tielens, 349

Cox, N. L. J., Kaper, L., \& Mokiem, M. R. 2005, A\&A, 436, 661

Cushing, M. C., Vacca, W. D., \& Rayner, J. T. 2004, PASP, 116, 362

D’Odorico, S., Dekker, H., Mazzoleni, R., et al. 2006, in SPIE Conf. Ser., 6269, 33

Ehrenfreund, P., Foing, B. H., d'Hendecourt, L., Jenniskens, P., \& Désert, F. X 1995, A\&A, 299, 213

Fitzgerald, M. P. 1970, A\&A, 4, 234

Foing, B. H., \& Ehrenfreund, P. 1994, Nature, 369, 296

Foing, B. H., \& Ehrenfreund, P. 1997, A\&A, 317, L59

Friedman, S. D., York, D. G., McCall, B. J., et al. 2011, ApJ, 727, 33

Galazutdinov, G. A., Krełowski, J., Musaev, F. A., Ehrenfreund, P., \& Foing, B. H. 2000, MNRAS, 317, 750

Geballe, T. R., Najarro, F., Figer, D. F., Schlegelmilch, B. W., \& de La Fuente, D. 2011, Nature, 479, 200

Gnacinski, P., Sikorski, J., \& Kaczmarczyk, G. 1997, Acta Astron., 47, 225
Gredel, R., Carpentier, Y., Rouillé, G., et al. 2011, A\&A, 530, A26 Groh, J. H., Damineli, A., \& Jablonski, F. 2007, A\&A, 465, 993

Herbig, G. H. 1993, ApJ, 407, 142

Herbig, G. H. 1995, ARA\&A, 33, 19

Hobbs, L. M., York, D. G., Thorburn, J. A., et al. 2009, ApJ, 705, 32

Jenniskens, P., \& Désert, F.-X. 1994, A\&AS, 106, 39

Joblin, C., d'Hendecourt, L., Léger, A., \& Maillard, J. P. 1990, Nature, 346, 729

Mattioda, A. L., Hudgins, D. M., \& Allamandola, L. J. 2005, ApJ, 629, 1188

Mazzei, P., \& Barbaro, G. 2011, A\&A, 527, A34

Modigliani, A., Goldoni, P., Royer, F., et al. 2010, in SPIE Conf. Ser., 7737, 28

Ruiterkamp, R., Cox, N. L. J., Spaans, M., et al. 2005, A\&A, 432, 515

Salama, F., Bakes, E. L. O., Allamandola, L. J., \& Tielens, A. G. G. M. 1996, ApJ, 458, 621

Salama, F., Galazutdinov, G. A., Krełowski, J., et al. 2011, ApJ, 728, 154

Sarre, P. J. 2006, J. Mol. Spectr., 238, 1

Snow, T. P. 2002, in 17th IAP Colloq. on Gaseous Matter in Galaxies and Intergalactic Space, eds. R. Ferlet, M. Lemoine, J.-M. Désert, \& B. Raban, 63

Snow, T. P., \& McCall, B. J. 2006, ARA\&A, 44, 367

Tielens, A. G. G. M. 2008, ARA\&A, 46, 289

Tuairisg, S. Ó., Cami, J., Foing, B. H., Sonnentrucker, P., \& Ehrenfreund, P. 2000, A\&AS, 142, 225

Vacca, W. D., Cushing, M. C., \& Rayner, J. T. 2003, PASP, 115, 389

Vernet, J., Dekker, H., D’Odorico, S., et al. 2011, A\&A, 536, A105

Vuong, M. H., \& Foing, B. H. 2000, A\&A, 363, L5

Watson, J. K. G. 2001, ApJ, 555, 472

Wegner, W. 1994, MNRAS, 270, 229

Zasowski, G., \& Ménard, B. 2014, in IAU Symp. 297, eds. J. Cami, \& N. L. J Cox, 68

Zasowski, G., Ménard, B., Bizyaev, D., et al. 2014, ApJ, submitted [arXiv: 1406.1195] 